\title{
Design, Testing and Performance Evaluation of Beam Positioning System for Free Space Optical Communication System
}

\author{
Tamilmani PASUPATHI, James ARPUTHA VIJAYA SELVI
}

Dept. of Electronics and Communication Engineering, Kings College of Engineering, Thanjavur, 613 303, India

pasu.tamil@gmail.com, randdece@gmail.com

Submitted September 25, 2020 / Accepted January 1, 2021

\begin{abstract}
Beam wandering and the wavefront distortion are the significant sources for the power loss in Wireless Optical Communication (WOC). In this paper Full Factorial Design (FFD) and Back Propagation Neural Network (BPNN) controller based autonomous beam monitoring, positioning and recovery system for fine steering of the laser beam at the focal point of the FSOC receiver is proposed. The proposed controllers process the intensity information of the received optical beam as inputs and produce the control signals as outputs. These control signals bring the beam at the focal point of the receiver and avoid the power loss of the optical link. The work describes performance analysis of Field Programmable Gate Array (FPGA) based novel digital architecture of FFD and $B P N N$ controller. Real time experimental verification of the stability and suitability of the developed adaptive controllers are tested for percentage of prediction error, Bit Error Rate (BER) and beam wander reduction ability and the same is demonstrated with suitable results. The experimental result shows that the BPNN controller gives high accurate approximation towards the control for the control signals $C_{x}$ and $C_{y}$ with the minimum and maximum values of $99.29 \%$ and $99.86 \%$ respectively. With the chosen parameters, the neuro-controller exhibits fast response for the error changes. The proposed BPNN controller provides prediction error very close to -0.5 to $+1.0 \%$, the values lie in the range of $-0.06781 \%$ and $0.9862 \%$ which shows that the BPNN controller is efficient for the real time tracking and control for FSOC, LIDAR imaging, micro/nano positioning, atomic force microscopes, scanning tunneling microscopes, etc.
\end{abstract}

\section{Keywords}

Optical communications, attenuation, opto-electronic, adaptive optics, neural network, beam steering

\section{Introduction}

FSOC attracts considerable interest for a large number of applications in telecommunications field due to its high data rate, low cost, free spectrum licensing, frequency coordination, interference free and fast installation. FSOC uses a visible Light Emitting Diode (LED), laser or invisible infrared for data communication which is similar to fiber optic communications. In FSOC beam of light, operating at very high frequency in the order of Terahertz region of the electromagnetic spectrum is collimated and transmitted over the atmosphere. Further, these are focused on a high sensitivity optical receiver through receiving telescope [1-3]. The random fluctuations in the atmosphere rigorously degrade the quality of the wavefront, thus resulting in intensity fluctuation and sometimes unavailability of the signal at the receiver end. In particular, beam wandering effect limits the use of the communication system. Incorporation of the beam steering system at the receiver end is the only possible solution to effectively mitigate and resolve the beam wandering and the received beam wavefront distortion effects respectively [4-8]. Mitigation of beam wandering (pointing error) to establish a perfect coupling of Power In Bucket (PIB): receiver aperture, to the communication optical detector plane becomes the most important as well as the very first essential need to effectively reduce the intensity fluctuation, beam outage and consequently recover the link quality and improve the overall performance of the FSOC system [9]. When using the FSOC link for fiber coupling, mobile and/or moving vehicle connectivity, it is necessary to align the optical axes of the both transmitting and receiving beam with sufficient accuracy and dynamic beam stabilization to improve the coupling efficiency and stability [10], [11].

Designing a suitable control scheme to minimize the beam displacement thereby significantly improving the beam stability and receiving power at the receiver detector plane becomes very necessary. To carry out this objective the experimental setups with the required optoelectronic components have been built for the transmission distance of $70 \mathrm{~m}$. In this paper adaptive controller design for beam steering is proposed using (i) Full Factorial Design (FFD) and (ii) Back Propagation Neural Network (BPNN) controller. Prediction error and focal point wander are considered as the performance metric.

The remainder of the paper is organized as follows: Section 2 presents the necessary backgrounds of the beam 
positioning system using different approaches, Section 3 briefs about FSOC opto-electronic test bed construction and error calculation unit, Section 4 describes the design of direct controller - FFD, Section 5 presents the efficient digital architecture of BPNN for beam monitoring, positioning and recovery, Section 6 discusses the analysis of results and validation of proposed schemes, Section 7 describes conclusion.

\section{Backgrounds and Related Works}

The problems associated with designing a closed loop control system for steering application and the works closely associated with the proposed work are described in this section. The importance of beam positioning system for FSOC is experimentally investigated for an indoor laboratory setup with turbulence simulation box and achieved BER of $10^{-9}$ [12]. Lu et al. proposed FPGA based optimized controller to mitigate the displacement error using high bandwidth stable precision steering controller. The structural response of the system is demonstrated with the integrated control simulation mechanism. Closed loop controller design and the hysteresis effect of the system are also described in [13]. Xie et al. described an adaptive controller using neural network approach. The controller consists of piezoelectric actuators with unknown hysteresis. Dynamic preinversion compensator is used to nullify the effect of the hysteresis [14]. A feedback controller for indoor steering system is demonstrated in [15]. Optical shaker is used to introduce the fluctuation in the optical beam and Fast Steering Mirror (FSM) is used to compensate the same. The system works on the PC based extended Kalman filter. In the studies of beam alignment/steering for FSOC systems, the main importances have been generally limited to the environment (indoor/outdoor) and mobility of the system.

Beam tilting mirrors and piezoelectric actuators based beam steering techniques are proposed for FSOC, visible light communication (VLC) and micro/nano manipulation control systems. Figure 1 illustrates basic beam steering system using mirrors in which the optical beam received at the receiver plane is steered by control signal. Piezoelectric/MEMS actuators are used to steer the beam in which perfect reflecting mirror is placed. This approach cannot be used in mobile devices due to a bulky receiver design and less accuracy. Figures 2(a) and (b) describe the different approaches of beam aligning systems using piezoelectric actuators. In this approach piezoelectric actuators are controlled by control signals generated through microcontrollers/FPGA. [16], [17].

The tilting approach in piezoelectric actuators has two different structures. In the first structure transmitter and receiver together are placed on piezoelectric actuators and in the later structure, only the lenses of the transmitter and receiver are placed on the actuators. In the implementation of a steering strategy for FSOC, one of the important problems is the presence of nonlinearity in the electronic/ piezoelectric actuators. This nonlinearity decreases the steer-

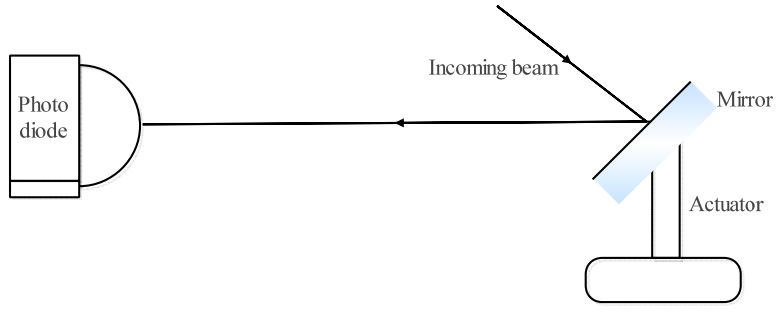

Fig. 1. Beam steering using mirrors.

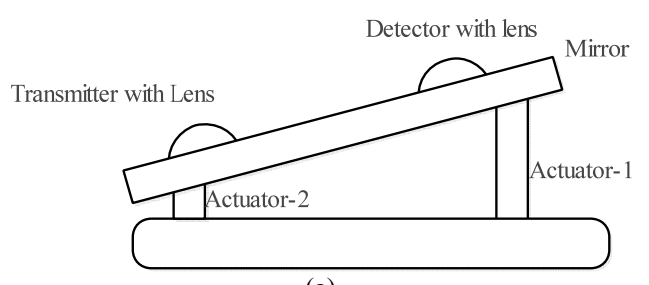

(a)

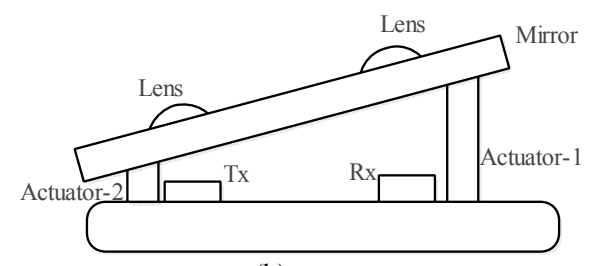

(b)

Fig. 2. (a) Transmitter and receiver placed on the actuators. (b) Only lenses placed on the actuators.

ing accuracy at the receiver unit. Few numbers of studies have been proposed to limit the drawback of nonlinear behavior in actuators using several types of controllers. Also most of the control system encountered in real time does not confirm to simple control configuration of Single Input-Single Output (SISO) variables. In the beam steering process, for example, beam stabilization cannot be done with a single control loop. These systems are intrinsically nonlinear which makes the controller design further difficult. The Artificial Neural Network (ANN) is the popular solution for nonlinearity due to its high performance, adaptivity and efficient input-output mapping to train from test/sample data and to provide solution to the trained or new data. Low-cost, low-power hardware implementation of steering controller becomes significant to handle the beam steering system at a very faster rate [18], [19]. Most of the real time experimental works describing the beam wandering mitigation process have been demonstrated with the laboratory setups built in the indoor atmosphere and the adaptive controllers are developed in the software environment and/or serial execution processors [20]. In this paper FFD \& BPNN controller is configured as a MIMO system for steering with two inputs and two output variables and this nonlinearity is greatly limited with the implementation of neural networks.

\section{FSOC Test Bed Construction and Error Computation Mechanism}

Figure 3 shows the complete schematic of the FSOC experimental setup using necessary opto-electronic assem- 
bly. Required digital architecture is developed for controlling the optical beam wandering of the optical beam. Pseudo Random Binary Sequence (PRBS) sequence generator in Non Return to Zero (NRZ) is used to generate the data in Mbps range at the transmitter. Semiconductor optical source $(850 \mathrm{~nm} / 50 \mathrm{~mW})$ is used as an optical source at the transmitter for establishing the FSOC link. Digital ASK (ON-OFF Keying) modulation is used as the optical modulator. The laser beam is modulated with $+5 \mathrm{~V} / \mathrm{DC}$ for binary ' 1 ' and $0 \mathrm{~V} / \mathrm{DC}$ for binary ' 0 '. Initially misalignment in the Line of Sight (LoS) is estimated and necessary corrections are made using of Line of Sight (Tx-Rx LoS) alignment controller. When the optical link is completely unavailable due to atmospheric turbulences or other factors, alignment controller at the receiver end immediately sends information to the alignment controller at the transmitter through RF transmitter/receiver for making the proper decision/alignment for establishing the optical communication. At the receiver end Newtonian type of telescope is used in the receiver side to collect the energy from the diverged laser beam. $91.66 \%$ of optical energy is being collected using the Newtonian telescope and the collected beam is collimated and passed to the Fast Steering Tip Tilt Mirror (FSTTM). The FSTTM is installed in the receiver experimental setup in order to steer the laser beam towards the desired co-ordinate based on the control signals from the FFD and BPNN based adaptive controller. Narrow Band Interference Optical Filter (NBIOF) allows $850 \mathrm{~nm}$ optical beam and rejects other unwanted radiations.

A circular type variable beam splitter (BS) is mounted on an optical bread board at $90^{\circ}$ angle to split the arrived beam into two directions. One part of the signal is made fall on the Avalanche Photo Diode (APD) for recovering the original information and to estimate the Bit Error Rate
(BER). The received data and estimated BER are displayed on the personal computer (PC). The second part of the signal also called as reflected beam from the variable BS falls on the 2 Dimensional-4 Quadrant (2D-4QD) detector that converts the incoming light into equivalent currents $I_{\mathrm{Q} 1}, I_{\mathrm{Q} 2}, I_{\mathrm{Q} 3}$ and $I_{\mathrm{Q} 4}$.

The output currents of the 2D-4QD are directly proportional to the intensity illuminating the detector surface. The angular displacements are measured with the relative changes in the output of 2D-4QD. The Mono-Pulse Arithmetic Circuit (MPAC) is used to measure the received optical signal intensity fluctuation and angular displacement. The output currents are applied into the MPAC that consists of I-V Converter Array, Error Acquisition Circuit (EAC) and Linear Level Shifter (LLS). The currents are linearly converted into relative voltage levels $V_{\mathrm{A}}, V_{\mathrm{B}}, V_{\mathrm{C}}$ and $V_{\mathrm{D}}$ by the I-V converter circuit array. The voltage outputs are connected to Error Acquisition Circuit (ECC) which consists of array of analog linear adder and subtractor.

The outputs of the ECC are $V_{\text {Ex }}, V_{\text {Ey }}$, and $V_{\text {Ref. The }}$ wandering of the arrived beam over the horizontal and vertical directions is calculated from $V_{\mathrm{Ex}}, V_{\mathrm{Ey}}$, respectively. The angular deflection of the arrived beam can be measured jointly by $V_{\mathrm{Ex}}, V_{\mathrm{Ey}}$. The intensity fluctuations due to atmospheric changes are measured by the output $V_{\text {Ref. The }}$ characteristics behavior of the error calculation circuit in $(x),(y)$ and $(x, y)$ directions are

$$
\begin{aligned}
& V_{\text {Ex }}=\left\{\left(V_{\mathrm{A}}+V_{\mathrm{C}}\right)-\left(V_{\mathrm{B}}+V_{\mathrm{D}}\right)\right\}, \\
& V_{\text {Ey }}=\left\{\left(V_{\mathrm{A}}+V_{\mathrm{B}}\right)-\left(V_{\mathrm{C}}+V_{\mathrm{D}}\right)\right\}, \\
& V_{\text {Ref }}=\left(V_{\mathrm{A}}+V_{\mathrm{B}}+V_{\mathrm{C}}+V_{\mathrm{D}}\right) .
\end{aligned}
$$

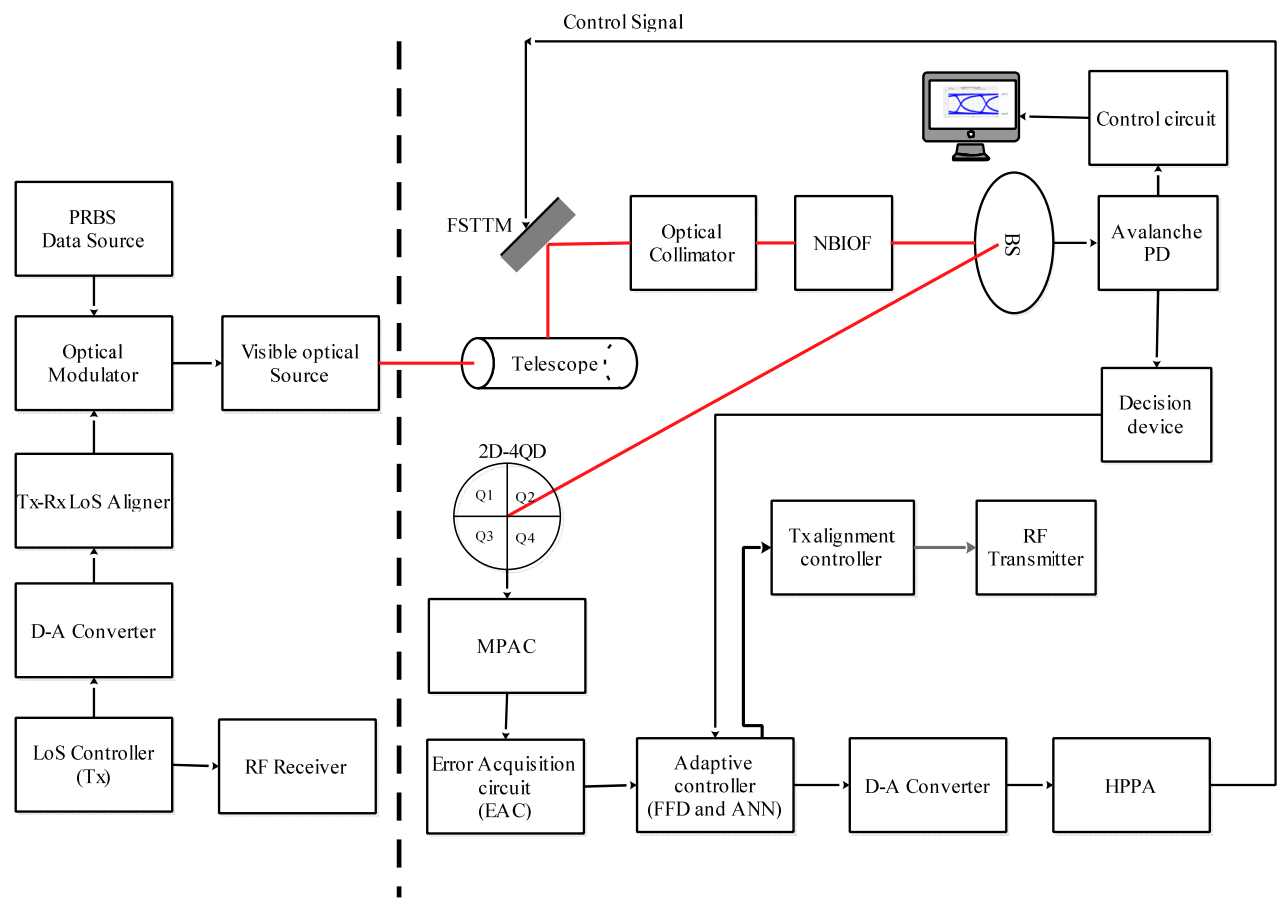

Fig. 3. Schematic of the FSOC experimental test bed (Transmitter and Receiver). 


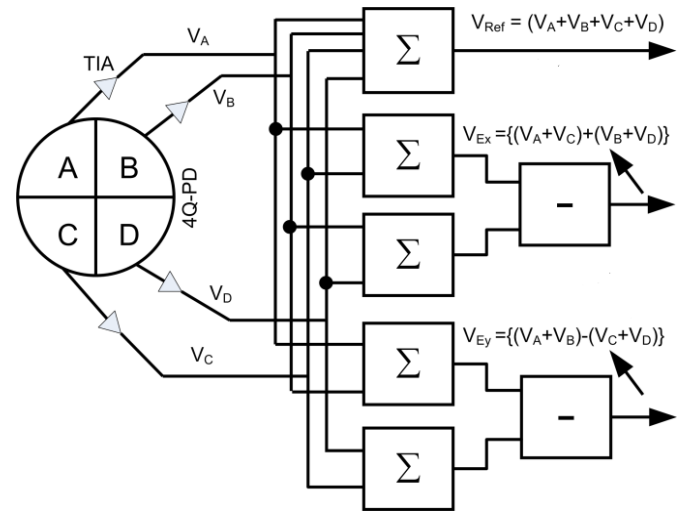

Fig. 4. Signal flow diagram and displacement error computation using MPAC.

The output of the error calculation circuit $V_{\mathrm{Ex}}, V_{\mathrm{Ey}}$ differs from $+12 \mathrm{~V}$ to $-12 \mathrm{~V}$ and $V_{\text {Ref }}$ differs from $0 \mathrm{~V}$ to $12 \mathrm{~V}$. Obtained voltage levels are shifted to $+2 \mathrm{~V}$ to $-2 \mathrm{~V}$ for interfacing the high speed A-D converter. These analog output signals $V_{\mathrm{Ex}}$ and $V_{\mathrm{Ey}}$ vary from $-12 \mathrm{~V}$ to $+12 \mathrm{~V}$ respectively as marked in Fig. 4.

The signals $V_{\mathrm{Ex}}, V_{\mathrm{Ey}}$ are also referred to as Process Variables (PVs). In both FFD and BPNN technique the outputs of 2D-4QD are applied to A-D converter and then the normalized error data are fed to the adaptive controller implemented in FPGA. The signal conditioning circuit is deployed in between FPGA and FSTTM. The Signal Conditioner Circuit (SCC) is constructed with 12-bit Digital to Analog Converter (DAC) and High Power Piezo Amplifier (HPPA) to process the servo control data generated by the adaptive controller. The main application of the SCC is converting digital to the piezo control output high voltage varies between $-20 \mathrm{~V}$ and $+120 \mathrm{~V}$. The DAC7728 is preferred due to the capability of parallel data input and high voltage output. The input data resolution is 12 bit and the output can either be a bipolar $\pm 16.5 \mathrm{~V}$ or unipolar $0 \mathrm{~V}$ to $+30 \mathrm{~V}$ with the reference voltage of $5.5 \mathrm{~V}$. The DAC 7728 is interfaced with FPGA through dedicated 14 GPIO pins. In the open loop mode, the $\mathrm{x}$ and $\mathrm{y}$ axis are controlled by $-7 \mathrm{~V}$ to $+7 \mathrm{~V}$.

\section{Design of FFD Controller and Performance Evaluation}

The experiments carried out for the established FSOC test bed using FFD based Response Surface Model (RSM) is described in this section. This model requires minimum knowledge about the process and target is achieved within fewer trails. This feature makes the system cost effective and less time consuming experimentation. Design and development of RSM model involves systematic planning and Design Of Experiments (DOE) approach of the experiments to be carried out. Initially DOE is used to identify the suitable experimental sampling points and, then these points are used as the ideal points for obtaining the response of the system. RSM based mathematical model for steering the actuators of the FSTTM is obtained by con-

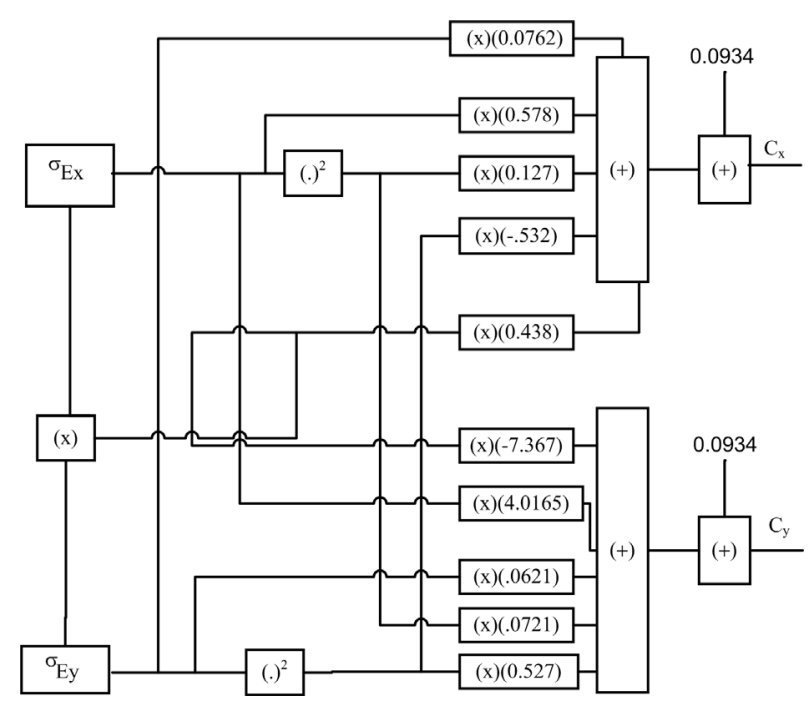

Fig. 5. Pipelined architecture of FFD controller implementation inside FPGA

\begin{tabular}{|c|c|c|c|c|c|c|}
\hline \multirow{3}{*}{$\begin{array}{c}\text { Trial } \\
\text { Expt } \\
\text { No }\end{array}$} & \multirow{2}{*}{\multicolumn{2}{|c|}{$\begin{array}{c}\text { Process } \\
\text { Variables [V] }\end{array}$}} & \multicolumn{4}{|c|}{ Predicted Control Variables } \\
\hline & & & \multicolumn{2}{|c|}{$\begin{array}{c}\text { Predicted Control } \\
\text { Value [V] }\end{array}$} & \multicolumn{2}{|c|}{$\begin{array}{c}\text { Predicted Contro] } \\
\text { Error }[\%]\end{array}$} \\
\hline & $V_{\mathrm{Ex}}$ & $V_{\mathrm{Ey}}$ & $C_{\mathrm{x}}$ & $C_{\mathrm{y}}$ & $C_{\mathrm{x}}$ & $C_{\mathrm{y}}$ \\
\hline 1 & 2.834 & 0.391 & 1.2412 & 3.690 & 8.9322 & 9.1281 \\
\hline 2 & 3.713 & 1.391 & -2.161 & -5.159 & 8.9757 & 8.9757 \\
\hline 3 & -3.012 & -2.931 & 6.004 & -4.060 & 2.5308 & 2.5308 \\
\hline 4 & -1.916 & 0.121 & -1.090 & -0.104 & 4.6857 & 4.6857 \\
\hline 5 & -3.674 & 2.631 & 2.564 & 0.531 & 7.3727 & 7.2646 \\
\hline 6 & 3.005 & 0.681 & -3.014 & -3.824 & -8.793 & 5.7562 \\
\hline 7 & -2.052 & -1.635 & 1.932 & 3.0051 & 7.3545 & 8.7421 \\
\hline 8 & 3.522 & 0.318 & 1.281 & 2.9563 & 5.8621 & 9.6533 \\
\hline
\end{tabular}

Tab. 1. Trial experiment design plan using FFD.

ducting trail experiments using FFD equation and described as follows,

$$
\begin{aligned}
C_{\mathrm{x}}= & 0.0934+0.578 V_{\mathrm{Ex}}+0.0672 V_{\mathrm{Ey}}+0.127 V_{\mathrm{Ex}}{ }^{2}+ \\
& 0.438 V_{\mathrm{Ex}} V_{\mathrm{Ey}}-0.0532 V_{\mathrm{Ey}}{ }^{2}, \\
C_{\mathrm{y}}= & -0.048+0.578 V_{\mathrm{Ex}}+0.062 V_{\mathrm{Ey}}+0.072 V_{\mathrm{Ex}}{ }^{2}- \\
& 7.367 V_{\mathrm{Ex}} V_{\mathrm{Ey}}-0.527 V_{\mathrm{Ey}}{ }^{2} .
\end{aligned}
$$

The pipelined architecture developed for the FFD controller using FPGA is shown in Fig. 5.

Initially the trail experiments are carried out for the established FSOC test bed. For each process variables ( $V_{\mathrm{Ex}}$ and $V_{\mathrm{Ey}}$ ) the control variables factors $C_{\mathrm{x}}$ and $C_{\mathrm{y}}$ are noted, and the prediction error is calculated. The above two equations are formulated by considering the individual and combined effects of input variables. Subsequent output is analyzed and mathematical equations are formulated.

The equations (4) and (5) are considered as a full model equation with three expressions (linear, quadratic and interaction) in terms of the process variables. The constants associated with the terms are called as regression coefficients. These coefficients are determined by,

$$
\mathbf{b}=\left(\mathbf{X}^{\mathrm{T}} \mathbf{X}\right)^{-1} \mathbf{X}^{\mathrm{T}} \mathbf{Y}
$$


where $\mathbf{b}$ represents the parameter estimate matrix, $\mathbf{X}$ and $\mathbf{Y}$ denotes calculation matrix and matrix of computed values of process variables respectively and $T$ represents transpose operator.

Table 1 shows the trail experimental design plan using FFD. The response surface of FFD controller clearly shows that the control signal for error input demonstrates nonlinear behavior and in addition, it is observed that percentage of predicted control errors for FFD controller varies with the value of $-8.793 \%$ and $9.6533 \%$.

\section{Design and Functionality of the BPNN Controller for Monitoring, Positioning and Recovery}

In recent years, the design of ANN controllers are developed by software environment and then realized in reconfigurable device like FPGA. In this paper FPGA is used for BPNN implementation due to its advantages of reconfigurability, low power consumption, parallelism and high speed computations [21], [22]. The design of neural-controller consists of more number of complex arithmetic computations, therefore, the weights and bias values of ANN controller are obtained from the software environment as a preliminary step for the execution of hardware. The computed weights, bias values along with VHDL code is compiled, synthesized and realized on Spartan-6 Xilinx FPGA with Xilinx ISE tool. The number of hidden layers and neurons used for designing neural-controller are decided through a series of trial and error method.

The design structure of BPNN controller using FPGA is shown in Fig. 6. FPGA implementation of neuro-controller uses the $100 \mathrm{MHz}$ internal clock frequency. Required frequencies for the operation of various units are synthesized by means of digital clock decimator design. The process variables $V_{\mathrm{Ex}}$ and $V_{\mathrm{Ey}}$ are normalized and available at the data register unit. Hidden layer1 (HL1) and hidden layer2 (HL2) computations are performed with necessary synaptic weight and bias values and are stored in the memory.

The neural-controller consists of 2-15-9-2 neurons in input, HL1, HL2 and output layer respectively. The log sigmoid function is used to model the bipolar sigmoidal neurons that are used in HL1 and HL2. Multiply and Accumulator Unit (MAC) multiplies the neurons with the corresponding synaptic weights and accumulated in memory. The synaptic weights of every neuron are stored in ROM memory. When one cycle of inputs are arrived, the output is added with suitable bias function. Figure 7 shows the one of the trained pattern with its necessary weights and bias.

A six stage back propagation neural network learning algorithm for the developed structure is summarized below:

Stage 1: Initialization of the neural network parameters: Initially the random weights and bias are assigned to all the neurons. The weights and bias are assigned with smaller random values; learning rate and goal of the network are also defined. In this work the neural network is trained for 1532 iterations with learning rate of 0.01 . The stopping criteria used for the network is $10^{-6}$.

Stage 2: Forward computation: The process variables $V_{\mathrm{Ex}}$ and $V_{\text {Ey }}$ are normalized and available at the input layer. The primitive functions and their derivatives are computed at each node and are stored. The computation performed by each neuron is as follows:

$$
o_{i}^{(m)}=g\left(H_{i}^{(m)}\right)=g\left(\sum_{j=1}^{S^{m+1}} w_{i, j}^{m} a_{j}^{m-1}+b_{i}^{m}\right)
$$

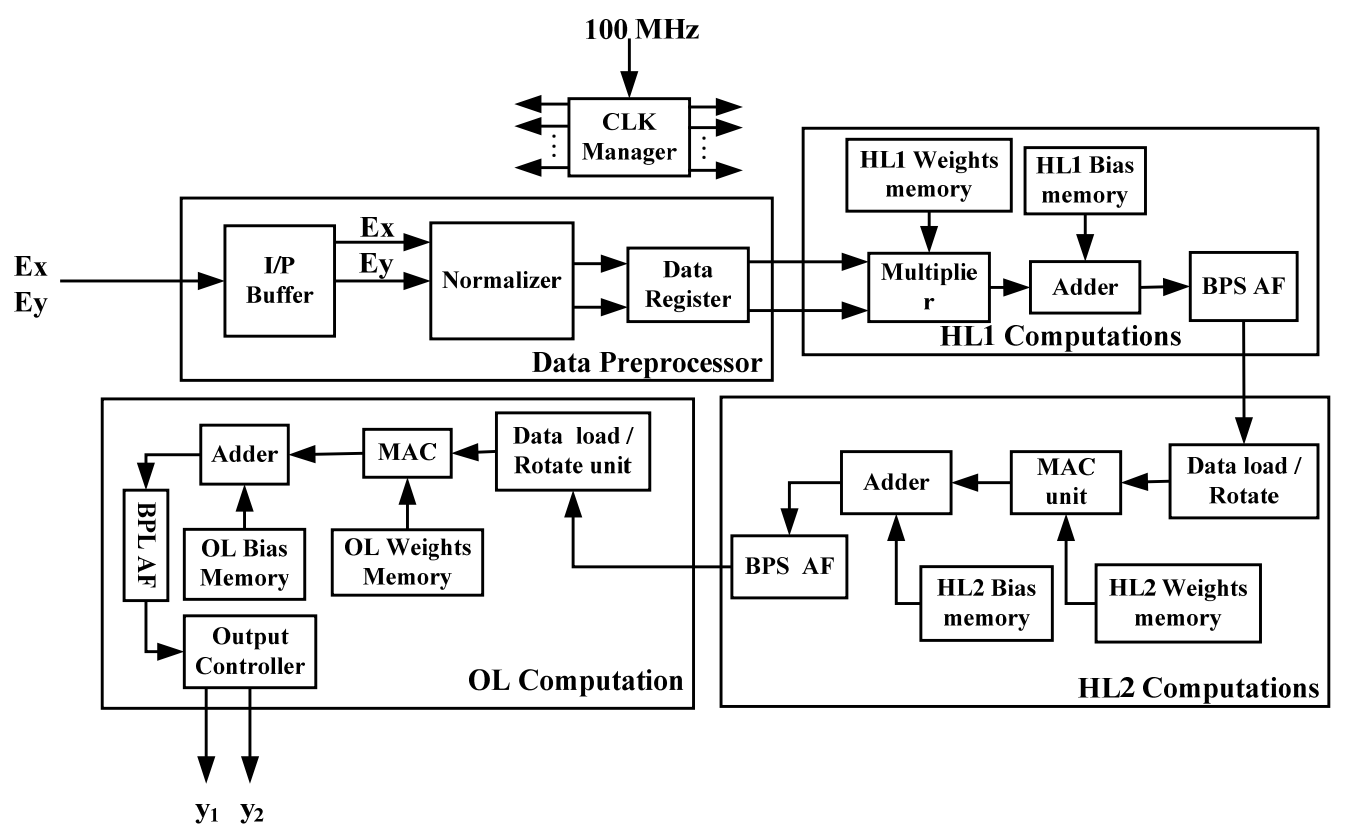

Fig. 6. Design structure of BPNN controller design using FPGA. 


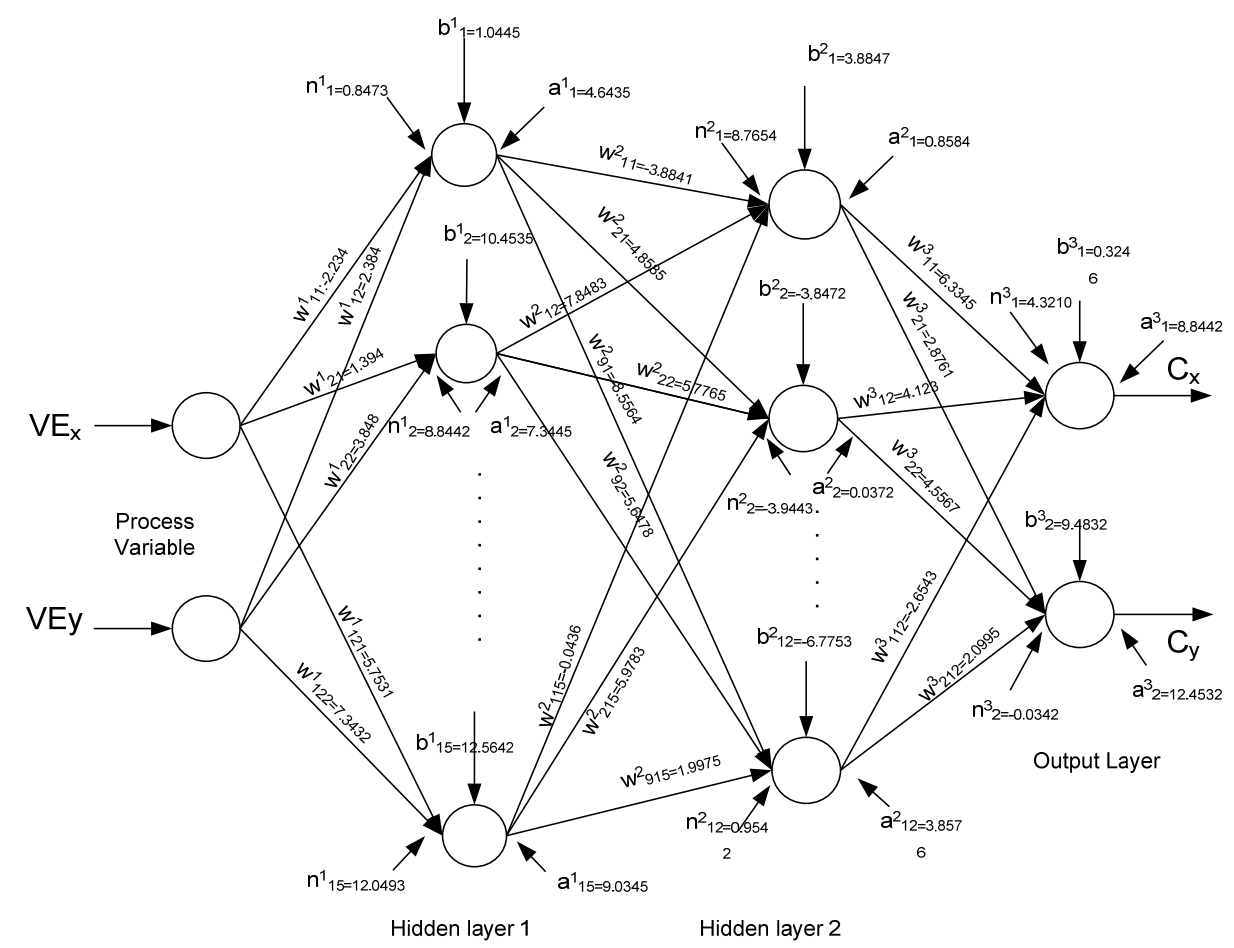

Fig. 7. Structure of BPNN - the trained pattern with its necessary weights and bias.

where $o_{i}{ }^{(m)}$ is neuron output, $H_{i}{ }^{(m)}$ is weighted sum, $w_{i, j}{ }^{m}$ : synaptic weight, $b_{i}{ }^{m}$ bias, $g\left(H_{i}^{(m)}\right)$ is activation function, $i=1,2,3, \ldots p-1, p$ and $m=1,2,3 \ldots \ldots \ldots q-1, q$.

Stage 3: Error term computation: The network is initially trained with well-known trail data and its output is computed. Actual output of the neural network model is computed during the real time experiment and an error function defined. The term $\xi_{i}^{(m)}$ is named as error term and calculated as follows,

$$
\xi_{i}^{(m)}=\left(t_{i}-o_{i}^{(m)}\right)
$$

where $t_{i}$ is the desired value from the $i^{\text {th }}$ neuron, and $o_{i}$ is the original output value of the $i^{\text {th }}$ neuron.

Stage 4: Backward computation: In this step the obtained error function is minimized till the desired goal is achieved by adjusting the weights of the neuron through back propagation. During this step the local gradients are calculated as follows

$$
\varepsilon_{i}^{(m)}=\xi_{i}^{(m)} g^{\prime}\left(H_{i}^{(m)}\right) .
$$

Stage 5: Updating weights: Weights of the neural network are adjusted to the best values according to the computation of the back propagation algorithm. The changes in weight and bias are calculated as follows

$$
\begin{gathered}
w_{i, j}^{m}(k+1)=w_{i, j}^{m}(k)-\alpha s_{i}^{m} \alpha_{j}^{m-1}, \\
b_{i}^{m}(k+1)=b_{i}^{m}(k)-\alpha s_{i}^{m}
\end{gathered}
$$

where $\alpha$ is learning rate.

Stage 6: Iterations for a desired convergence: Learning rate, stopping criteria and the optimization method used are the important parameters of deciding number iterations of neural network. During every iteration, the local gradient descents are updated with necessary weights and minimum global loss function. Finally the best one for the optimum control signal is estimated and the values are stored in the memory.

\section{Validation of FFD and BPNN Model}

Initial position of the beam must be set to $(0,0)$ for the proposed controllers to get the desired response. Large numbers of trail experiments are carried out to confirm the performance of the FFD and BPNN controllers. Coefficient of determination $\left(R^{2}\right)$ values obtained from the FFD and BPNN control signals are listed in Tab. 2. $R^{2}$ value is a statistical measure used to analyze how differences in one variable with respect to difference in another variable also it shows how close the measured data are to the fitted regression line. $R^{2}$ value for the full model equation of the FFD controller gives $C x$ and $C y, 91.54 \%$ and $92.91 \%$, respectively. Whereas, the BPNN controller provides necessary approximation towards the control of beam wandering with the minimum and maximum values of $99.29 \%$ and $99.86 \%$. The computed variable for $C_{\mathrm{x}}$ and $C_{\mathrm{y}}$ clearly shows that the linear $\left(V_{\mathrm{Ex}}, V_{\mathrm{Ey}}\right)$, quadratic $\left(V_{\mathrm{Ex}}{ }^{2}, V_{\mathrm{Ey}}{ }^{2}\right)$ and interaction $\left(V_{\mathrm{Ex}} \times V_{\mathrm{Ey}}\right)$ terms are the major contributing parameter for controlling horizontal and vertical displacement of the optical beam. For the desired horizontal displacement control the contribution of $V_{\mathrm{Ex}}$ is $58.54 \%$ and $V_{\mathrm{Ey}}$ is $3.815 \%$ and for the desired vertical displacement control the contribution of $V_{\mathrm{Ey}}$ is $53.14 \%$ and $V_{\mathrm{Ex}}$ is $2.472 \%$. The effect of $V_{\mathrm{Ex}}{ }^{2}$ and $V_{\mathrm{Ey}}{ }^{2}$ are extremely small towards the desired control. Confirmatory tests are used for the consid- 
eration of full model and BPNN controller. Also the performance of the FFD controller with full model was tested with higher polynomials such as the 3rd order and 4th order; however the performance of the model reached the saturation at higher order polynomials. Therefore the proposed equation (4) and (5) in the reported model is finalized and used for the control operation.

The real time experiments demonstrate that the BPNN controller can effectively compensate the displacement error by up to $99.86 \%$ and the tracking performance of reference control signal is significantly great. The entire reference output can be covered by mapping the input-output characteristics from the 741 training patterns. The desired output variables are generated progressively without overshoot when input signals $\left(V_{\mathrm{Ex}}\right.$ and $\left.V_{\mathrm{Ey}}\right)$ are injected with random value. The input is limited between $-12 \mathrm{~V}$ to $+12 \mathrm{~V}$, whereas the output is $-7 \mathrm{~V}$ to $+7 \mathrm{~V}$ and sampling period is $0.1 \mathrm{sec}$. With the chosen parameters, the neurocontroller exhibits fast response for the error changes. The goal of this method is to explore the capability of the neuro-controller to follow the reference profile as closely as possible at the fast rate. The proposed BPNN controller generates the response very close to the target values. The percentage of error predicted under FFD and BPNN controller are estimated and given in Tab. 3.

The plot of percentage of prediction error using FFD and BPNN controller experiment is shown in Fig. 8. The percentage of prediction errors estimated for FFD controller and the values lie in the range of $4.393 \%$ and $8.2531 \%$. BPNN controller provides prediction error very close to -0.5 to $+1.0 \%$, the values lie in the range of $0.06781 \%$ and $0.9862 \%$.

The stability of beam (X and $\mathrm{Y}$ axis) under FFD and BPNN controllers at the receive plane and a portion of data is shown in Fig. 9(a) and (b). Optimum control and tracking of the signal is achieved under BPNN controller in both $\mathrm{X}$ and $\mathrm{Y}$ axis. The average min-max and standard values of -4.922 to $+4.123 \mathrm{~mm}$ and $0.927 \mathrm{~mm}$ respectively in the FFD control and -1.884 to +2.072 and $0.025 \mathrm{~mm}$ respectively in BPNN control.

The beam displacement position under FFD and BPNN controllers at the receive plane and a portion of data are shown in Figs. 10(a), (b). The following observations

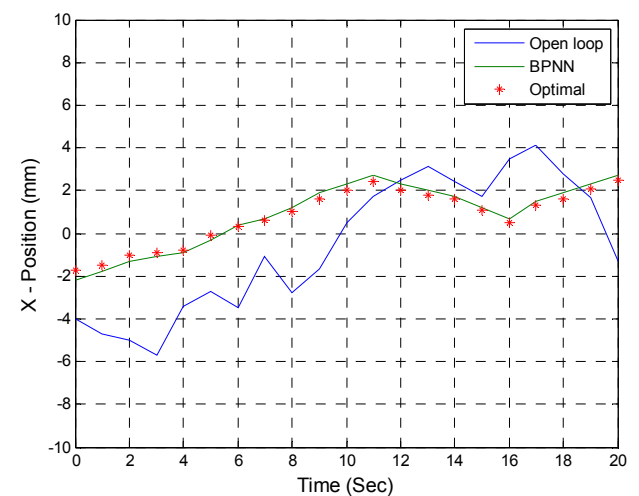

(a) are made under FFD controller design: (i) Beam wanders entire area of the detector surface. (ii) Movements of the beam are random and not predictable. (iii) Power fluctuation at the receiver plane is more. (iv) Sometimes, the beam goes out-off detector. During the time optical link is completely disconnected. Under the control of BPNN controller: (i) Movement of the beam is entirely controlled. (ii) Maximum intensity and power level is achieved. (iii) Optical link disconnectivity due to wandering of the beam is completely reduced.

\begin{tabular}{|c|c|c|c|c|c|c|}
\hline \multirow{3}{*}{ 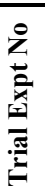 } & \multirow{2}{*}{\multicolumn{2}{|c|}{$\begin{array}{c}\text { Process } \\
\text { variables }[V]\end{array}$}} & \multicolumn{4}{|c|}{ Accuracy (\%) } \\
\hline & & & \multicolumn{3}{|c|}{ FFD controller } & \multirow[b]{2}{*}{ BPNN } \\
\hline & $\mathbf{V}_{\mathbf{E x}}$ & $\mathbf{V}_{\mathbf{E y}}$ & Linear & $\begin{array}{l}\text { Inter } \\
\text { action }\end{array}$ & Quadratic & \\
\hline 1 & 2.83 & 0.39 & 91.54 & 91.54 & 91.63 & 99.63 \\
\hline 2 & 3.71 & 1.39 & 90.34 & 90.34 & 91.58 & 99.58 \\
\hline 3 & -3.01 & -2.93 & 91.09 & 90.23 & 90.29 & 99.29 \\
\hline 4 & -1.91 & 0.12 & 92.04 & 92.51 & 92.71 & 99.71 \\
\hline 5 & -3.67 & 2.63 & 91.54 & 92.91 & 91.63 & 99.86 \\
\hline
\end{tabular}

Tab. 2. Accuracy approximation of FFD and BPNN controller.

\begin{tabular}{|c|c|c|c|c|c|c|c|}
\hline \multicolumn{3}{|c|}{ FFD controller } & \multicolumn{4}{c|}{ BPNN controller } \\
\hline $\begin{array}{c}\text { Predicted value } \\
{[V]}\end{array}$ & \multicolumn{2}{|c|}{$\begin{array}{c}\text { Prediction } \\
\text { error [\%] }\end{array}$} & \multicolumn{2}{c|}{$\begin{array}{c}\text { Predicted } \\
\text { value [V] }\end{array}$} & \multicolumn{2}{c|}{$\begin{array}{c}\text { Prediction } \\
\text { error [\%] }\end{array}$} \\
\hline$C_{\mathrm{x}}$ & $C_{\mathrm{y}}$ & $C_{\mathrm{x}}$ & $C_{\mathrm{y}}$ & $C_{\mathrm{x}}$ & $C_{\mathrm{y}}$ & $C_{\mathrm{x}}$ & $C_{\mathrm{y}}$ \\
\hline 1.35 & 3.21 & 8.39 & -9.19 & 1.24 & 3.69 & 0.01 & 0.00 \\
\hline 2.56 & -5.33 & 2.70 & -2.39 & 2.16 & -5.16 & 0.00 & 0.02 \\
\hline 6.11 & -4.32 & 4.14 & -8.00 & 6.01 & -4.05 & 0.01 & 0.00 \\
\hline 5.43 & -3.32 & 4.32 & -7.43 & 5.94 & 3.54 & 0.01 & 0.03 \\
\hline
\end{tabular}

Tab. 3. Validation test results of FFD and BPNN controller for $C_{\mathrm{x}}$ and $C_{\mathrm{y}}$.

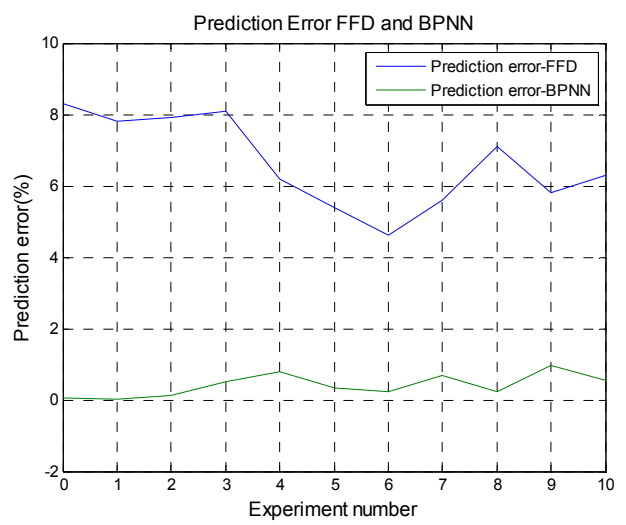

Fig. 8. Prediction of error under FFD and BPNN controller.

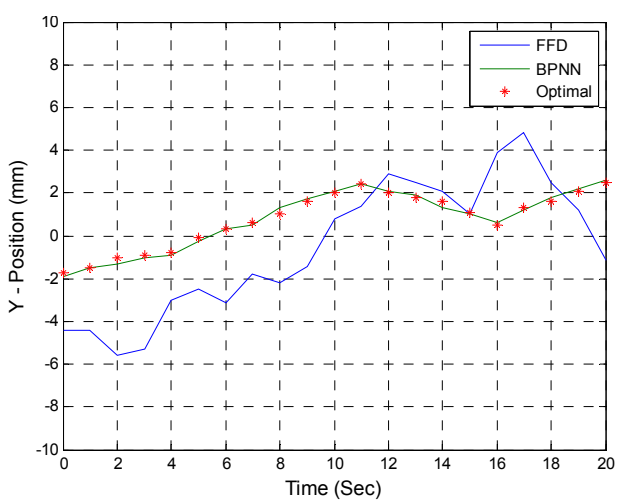

(b)

Fig. 9. (a) Beam stability (X-axis) under FFD and BPNN controller. (b) Beam stability (Y-axis) under FFD and BPNN controller. 

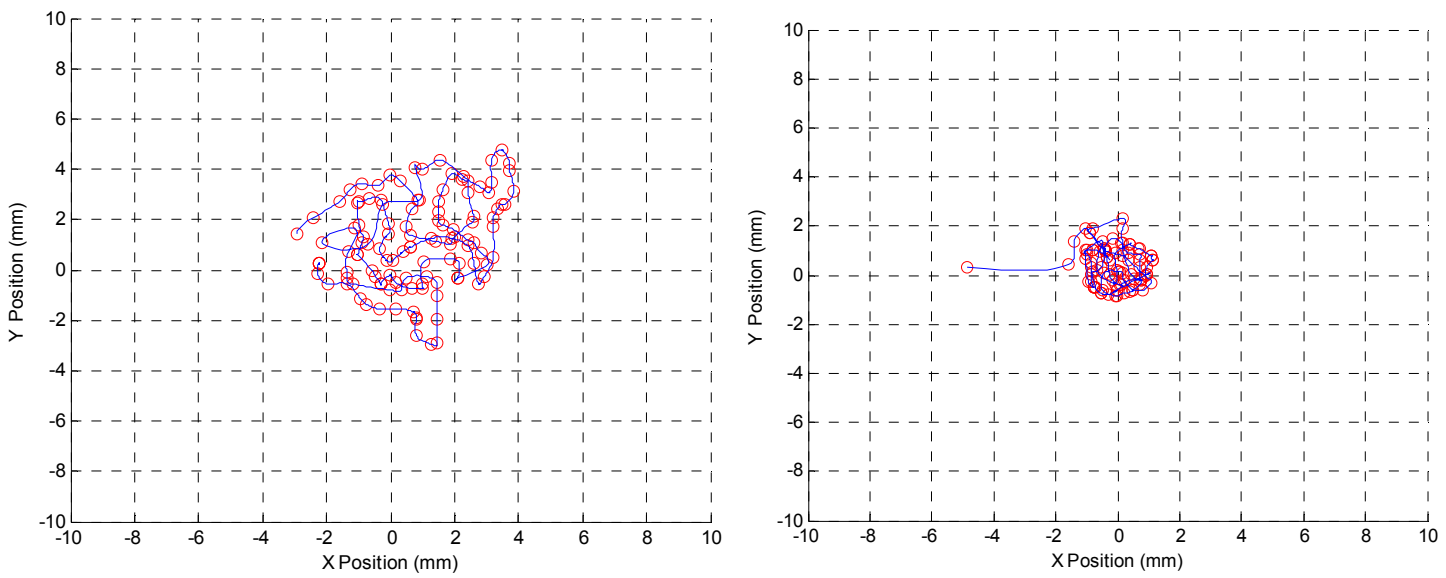

Fig. 10. (a) Displacement of the beam spot under FFD controller. (b) Displacement of the beam spot under BPNN controller.

\begin{tabular}{|c|c|c|c|c|c|c|c|c|}
\hline \multirow{2}{*}{\multicolumn{2}{|c|}{$\begin{array}{l}\text { Process variables } \\
{[\text { [V] }}\end{array}$}} & \multicolumn{4}{|c|}{$\begin{array}{c}\text { Control variables } \\
\end{array}$} & \multicolumn{2}{|c|}{ BER } & \multirow{3}{*}{$\begin{array}{c}\text { Control signal for enhancing power } \\
\text { at transmitter }\end{array}$} \\
\hline & & \multicolumn{2}{|c|}{$\begin{array}{c}\text { Trial experiment } \\
{[\mathrm{V}]}\end{array}$} & \multicolumn{2}{|c|}{$\begin{array}{c}\text { BP Neural-Network } \\
{[\text { V] }}\end{array}$} & \multirow[t]{2}{*}{ FFD } & \multirow[t]{2}{*}{ BPNN } & \\
\hline$V_{\mathrm{Ex}}$ & $V_{\mathrm{EV}}$ & $R_{\mathrm{x}}$ & $R_{\mathrm{y}}$ & X-tilt $\left(C_{x}\right)$ & Y-tilt $\left(C_{\mathrm{y}}\right)$ & & & \\
\hline 2.8342 & 0.3912 & 1.2412 & 3.690 & 1.3288 & 3.0234 & $2.3502 \times 10^{-3}$ & $4.6709 \times 10^{-8}$ & Perfect link, No changes \\
\hline 3.7137 & 1.3915 & -2.1612 & -5.159 & -1.132 & -5.760 & $1.0934 \times 10^{-2}$ & $3.7892 \times 10^{-9}$ & Perfect link, No changes \\
\hline-3.012 & -2.931 & 6.0045 & -4.060 & 4.0169 & -4.059 & $2.2905 \times 10^{-3}$ & $8.034 \times 10^{-8}$ & Poor link, Power level to be increased \\
\hline-1.914 & 0.1217 & -1.0903 & -0.104 & -1.032 & -0.104 & $3.5078 \times 10^{-4}$ & $7.892 \times 10^{-9}$ & Poor link, Power level to be increased \\
\hline
\end{tabular}

Tab. 4. Link quality measurement and comments on respective control signal.

The BPNN controller gives exact corrective action over the FFD controller to reduce the wandering of the beam. Link quality measurement and respective control signal is passed to transmitter for enhancing the power level is given in Tab. 4 for several trial experiments. The performance improvement of beam stability and link availability are tested under FFD and BPNN controller. Very controlled BER in the range of $3.78 \times 10^{-9}$ to $7.89 \times 10^{-9}$ is achieved in all the fluctuations with effective mitigation of the beam wandering under BPNN controller.

The developed model is optimized to the sufficient latency control action. Since the atmosphere disturbance is of very low frequency which is in the order of Hz. Control action of the developed controller is operated at several $\mathrm{kHz}$ speed which is more than sufficient to bring the tilted beam into the centroid of the optical position detector. In our experiment, the measured maximum latency is $0.18 \mathrm{~ms}$ and the average latency is $0.193 \mathrm{~ms}$.

\section{Conclusion}

A novel digital pipelined-parallel architecture developed for the hardware implementation of FFD and BPNN controller in the FPGA is discussed in this paper. The capability of the proposed controllers for the position information observability and the generation of corresponding control signal are tested. These results are recorded and compared against the control signal obtained in the trial experiment. The average min-max and standard values of -1.52 to $+1.32 \mathrm{~mm}$ and $0.327 \mathrm{~mm}$ respectively in the FFD control and -0.084 to +0.072 and $0.025 \mathrm{~mm}$ respectively in BPNN control are achieved for both the channels. Maximum efficiency of control response is attained using the
BPNN controller configuration. The results presented in this paper are intended for setting up the FSO communication transceiver. Therefore, it is suggested that this approach has lot of advantages and can be used for fine steering applications including monitoring, positioning and recovery.

\section{References}

[1] BOFFI, P., PICCININ, D., MOTTARELLA, D., et al. All-optical free-space processing for optical communication signals. Optics Communications, 2007, vol. 181 , no. $1-3$, p. 79-88. DOI: 10.1016/S0030-4018(00)00745-8

[2] HENNIGER, H., WILFERT, O. An introduction to free-space optical communications. Radioengineering, 2010, vol. 19, no. 2, p. 203-212. ISSN 1210-2512

[3] AROCKIA BAZIL RAJ, A., LANCELOT, J. P. Seasonal investigation on prediction accuracy of atmospheric turbulence strength with a new model at Punalkulam, Tamil Nadu. Journal of Optical Technology, 2015, vol. 83, no. 1, p. 55-68. DOI: 10.1364/JOT.83.000055

[4] MAJUMDAR, A. K. Advanced Free Space Optics (FSO): A Systems Approach. New York: Springer, 2015. DOI: 10.1007/978-1-4939-0918-6

[5] TAKAHASHI, K. Next Generation Optical Wireless Communication Systems Using Fiber Direct Coupled Optical Antennas. Chapter in: Das, N. (Ed.) Optical Communication. [Online] Available at: www.intechopen.com. DOI: 10.5772/48395

[6] FU, F., ZHANG, B. The influence of high-frequency phase distortion on the phase correction effect in atmosphere. Optik, 2014, vol. 125, no. 1, p. 360-365. DOI: 10.1016/j.ijleo.2013.06.046

[7] MUTAFUnGWA, E., HALME, S. J., KAZAURA, K., et al. Millimeter-wave over fiber systems using hybrid OCDM/WDM transmission. International Journal of Infrared and Millimeter 
Waves, 2003, vol. 24, p. 1113-1126. DOI: 10.1023/A:1024640200525

[8] SHARIFI, M., WU, G., LUO, B., et al. Beam wander of electromagnetic partially coherent flat-topped beam propagating in turbulent atmosphere. Optik, 2014, vol. 125, no. 1, p. 561-564. DOI: $10.1016 / j$. .jleo.2013.07.025

[9] MITCHELL, P. V. Fast Steering Mirror Technology: Active Beam Stabilization. Application note. Newport Corporation, USA 7 pages. [Online] Available at: https://www.newport.com/medias/sys_master/images/images/h4b/ h31/8797093363742/Fast-Steering-Mirror-Technology-App-Note2.pdf. ISBN: 01012 (01-01)

[10] CIZMAR, J., NEMECEK, J. Design and modeling of the properties of the servomechanism for a mobile free space optical link. Radioengineering, 2014, vol. 23, no. 1, p. 468-473. ISSN $1210-2512$

[11] UR REHMAN, S., ULLAH, S., CHONG, P. H. J., et al. Visible light communication: A system perspective-overview and challenges. Sensors, 2019, vol. 19, no. 5, p. 1-22. DOI: $10.3390 / \mathrm{s} 19051153$

[12] LIU, W., SHI, W., CAO, J., et al. Bit error rate analysis with realtime pointing errors correction in free space optical communication systems. Optik, 2014, vol. 125 , no. 1, p. 324-328. DOI: $10.1016 /$ j.ijleo.2013.06.043

[13] LU, Y., FAN, D., ZHANG, Z. Theoretical and experimental determination of bandwidth for a two-axis fast steering mirror Optik, 2013, vol. 124, no. 16, p. 2443-2449. DOI: 10.1016/j.ijleo.2012.08.023

[14] XIE, W., FU, J., YAO, H., et al. Neural network based adaptive control of piezoelectric actuator with unknown hysteresis. Adaptive Control and Signal Processing, 2009, vol. 23, no. 1, p. 30-54. DOI: $10.1002 /$ acs. 1042

[15] PEREZ-ARANCIBIA, N. O., GIBSON, J. S., TSAO, T. Observerbased intensity-feedback control for laser beam pointing and tracking. IEEE Transactions on Control System Technology, 2012, vol. 20, no. 1, p. 31-47. DOI: 10.1109/TCST.2011.2109720

[16] ZHANG, Q., ZHAO, J., SHEN, X., et al. Modeling, and testing of a novel XY piezo-actuated compliant micro-positioning stage. Micromachines, 2019, vol. 10, no. 9, p. 1-19. DOI: 10.3390/mi10090581

[17] CAO, Z., ZHANG, X., OSNABRUGGE, G., et al. Reconfigurable beam system for non-line-of-sight free-space optical communication. Light: Science \& Applications, 2019, vol. 8, p. 1-9. DOI:10.1038/s41377-019-0177-3

[18] SPRANGLE, P., TING, A., PENANO, J., et al. Incoherent combining and atmospheric propagation of high-power fiber lasers for directed-energy applications. IEEE Journal of Quantum Electronics, 2009, vol. 45, no. 2, p. 138-148. DOI: 10.1109/JQE.2008.2002501

[19] YUKSEL, M., AKELLA, J., KALYANARAMAN, S., et al. Freespace-optical mobile ad hoc networks: Auto-configurable building blocks. Wireless Networks, 2009, vol. 15, p. 295-312. DOI: 10.1007/s11276-007-0040-y
[20] FIElhaueR, K. B., BOONE, B. G., BRUZZI, J. R., et al. Comparison of macro-tip/tilt and meso-scale position beam steering transducers for a free space optical communications using a quadrant photodiode sensor. In SPIE's 48th Annual Meeting: Optical Science and Technology. Diego (CA, USA), 2003. DOI: $10.1117 / 12.506179$

[21] SHOME, S. K., MUKHERJEE, A., KARMAKAR, P., et al. Adaptive feed-forward controller of piezoelectric actuator for micro/nano-positioning, Sadhana, 2018, vol. 43, no. 158, p. 1-9. DOI: $10.1007 / \mathrm{s} 12046-018-0925-8$

[22] LE, Q. N., JEON, J. W. Neural-network-based low-speed-damping controller for stepper motor with an FPGA. IEEE Transactions on Industrial Electronics, 2010, vol. 57, no. 9, p. 3167-3180. DOI: 10.1109/TIE.2009.2037650

\section{About the Authors ...}

Tamilmani PASUPATHI was born in India. He received his Bachelor of Engineering in Electronics and Communication Engineering from Anna University, and Master of Engineering in VLSI Design from Anna University in 2012. He joined in Laser Communication Laboratory of Kings College of Engineering in 2012 and involved in research project titled Mono-Pulse and Artificial Neural Network Based Atmospheric Turbulence Effects Mitigation in Terrestrial Free Space Line of Sight Optical Communication (TFSLoSOC) System (No: ERIP/ER/0704390/ $\mathrm{M} / 01 / 1102)$. His current research interests are wireless optical communication, digital design using FPGA.

James ARPUTHA VIJAYA SELVI was born in India. She received the Bachelor of Engineering in Electronics and Communication Engineering from the Government College of Engineering, Thirunelveli, India, both M.E degree in CS and Ph.D. degree from the National Institute of Technology (NIT), Tiruchirappalli, India. She served for 9 years as the faculty in the ECE department at SASTRA University, Thanjavur, India. She headed several research projects funded by MC\&IT, DRDO, AICTE, CSIR, ISTE New Delhi and TNSTC - Chennai. She has received best teacher and best paper awards. Since April 2006, she has been the professor at the Kings College of Engineering, Thanjavur. Her research interests are in the broad areas of communication theory and networking, empirical modeling, performance evaluation of wired / wireless communication systems, adaptive optics, process control, image processing, neural networks. She has published more than 30 papers in various reputed national and international journals. 